Supplementary Material for

\title{
Novel Ozone-Mediated Cleavage of the Benzhydryl Protecting Group from Aziridinyl Esters
}

\author{
Aniruddha P. Patwardhan, Zhenjie Lu, V. Reddy Pulgam and William D. Wulff* \\ Department of Chemistry \\ Michigan State University \\ East Lansing, MI 48824
}

\section{General Information}

All chemicals were purchased from Aldrich. All solvents were reagent grade and were distilled under argon before use: dichloromethane $\left(\mathrm{CH}_{2} \mathrm{Cl}_{2}\right)$, toluene and benzene from calcium hydride $\left(\mathrm{CaH}_{2}\right)$, tetrahydrofuran and diethyl ether from benzophenone ketyl. Ozonolysis was performed on a Welsbach T23 ozonoator at a flow rate of $0.0965 \mathrm{scfm}$ (standard cubic feet per min) of air. NMR spectra were recorded on a Varian $300 \mathrm{MHz}$ spectrometers using ${ }^{1} \mathrm{H}$ and ${ }^{13} \mathrm{C}$ solvent peaks as an internal reference (e.g., $7.240 \mathrm{ppm}$ for $\mathrm{CDCl}_{3}$ and the $\mathrm{CDCl}_{3}$ triplet at $77.00 \mathrm{ppm}$ ). FT-IR were recorded on a Nicolet IR/42 spectrometer. Both low and high resolution mass spectra were obtained from the Michigan State University Mass Spectrometry Facility. Elemental analyses were done by the Michigan State University Elemental Analysis Facility.

\section{General experimental procedure for the deprotection via hydrogenolysis illustrated for $\mathbf{3 b}$.}

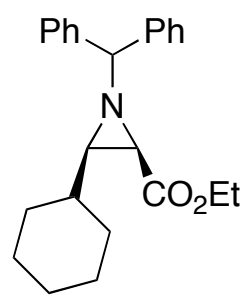

3b

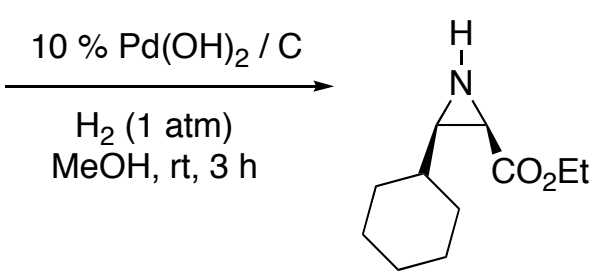

6b $93 \%$

cis-(2R)-Carboxyethyl-(3R)-cyclohexylaziridine (6b): To a $250 \mathrm{~mL}$ flame-dried round bottom flask filled with argon was added $363 \mathrm{mg}$ cis-N-benzylhydryl-2-carboxyethyl-3cyclohexylaziridine $(1 \mathrm{mmol}), 170 \mathrm{mg} \mathrm{Pd}(\mathrm{OH})_{2}(0.1 \mathrm{mmol})\left(\mathrm{Pd}(\mathrm{OH})_{2}\right.$ on carbon $20 \%$, moisture ca $60 \%$ ) and $80 \mathrm{~mL} \mathrm{MeOH}$. The flask was equipped with a vacuum transfer adapter connected to 
vacuum and a balloon filled with hydrogen. The valve to vacuum was opened for a few seconds, then switched to the hydrogen balloon, this process was repeated for 4 times. The suspension was stirred at r.t under hydrogen for $4 \mathrm{~h}$ and filtered through a pad of Celite, washed with ether $(20 \mathrm{~mL})$ and the filtrate was concentrated by rotary evaporation, followed by flash chromatography (15 $\mathrm{mm}$ $\times 300 \mathrm{~mm}$ silica gel, 1:1 ether/hexane) to afford $\mathbf{6 b}$ as a white solid in $93 \%$ yield (183 $\mathrm{mg})$. White solid: mp: $58-59{ }^{\circ} \mathrm{C} ; \mathrm{R}_{f}=0.19$ (1:1 Et $\mathrm{Et}_{2} \mathrm{O}$ hexane). Spectral data for $\mathbf{6 b}:{ }^{1} \mathrm{H}-\mathrm{NMR}\left(\mathrm{CDCl}_{3}, 300 \mathrm{MHz}\right)$ $\delta=0.90($ br s, $1 \mathrm{H}), 1.05-1.22(\mathrm{~m}, 6 \mathrm{H}), 1.24(\mathrm{t}, \mathrm{J}=7.1 \mathrm{~Hz}, 3 \mathrm{H}), 1.40-1.45(\mathrm{~m}, 1 \mathrm{H}), 1.52-1.70(\mathrm{~m}$, $3 \mathrm{H}), 1.80-2.00(\mathrm{~m}, 2 \mathrm{H}), 2.60(\mathrm{~d}, \mathrm{~J}=6.1 \mathrm{~Hz}, 1 \mathrm{H}), 4.18(\mathrm{q}, \mathrm{J}=7.1 \mathrm{~Hz}, 2 \mathrm{H}) ;{ }^{13} \mathrm{C}\left(\mathrm{CDCl}_{3}, 75 \mathrm{MHz}\right) \delta=$ 14.14, 25.39, 25.40, 26.01, 30.84, 31.61, 34.24, 36.91, 43.98, 61.06, 170.95; IR (thin film, $\mathrm{cm}^{-1}$ ) 3165(w), 2924(vs), 2849(s), 1730(vs), 1194(s), 1180(s); mass spectrum, $\mathrm{m} / \mathrm{z}$ (\% rel intensity) 197 $\mathrm{M}^{+}(4), 168$ (32), 124 (100), 95 (84). Anal calcd for $\mathrm{C}_{11} \mathrm{H}_{19} \mathrm{NO}_{2}$ : C, 66.97; H, 9.71; N, 7.10. Found: C, 66.98; H, 9.30; N, 7.08.

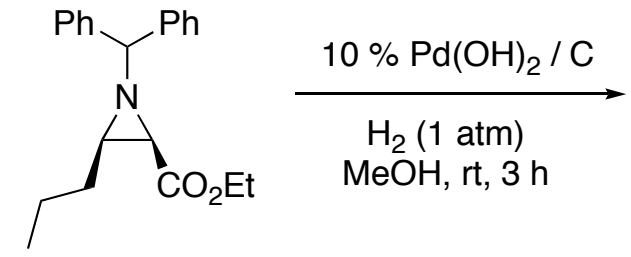

$3 a$

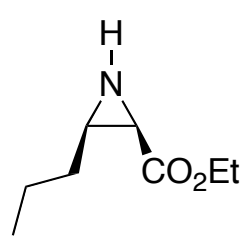

6a $83 \%$

cis-(2R)-Carboxyethyl-(3R)- $\boldsymbol{n}$-propylaziridine (6a): This reaction was carried out with the general procedure with a slight change in the purification. The product evaporates if rotary evaporation is used to remove the solvent after chromatography, so the eluent was adjusted to 1:1 ether/ pentane and distillation was used to remove the solvent. The aziridine $\mathbf{6 a}$ was afforded as a colorless liquid in $83 \%$ yield $(130 \mathrm{mg}) . \mathrm{R}_{f}=0.13\left(1: 1 \mathrm{Et}_{2} \mathrm{O} /\right.$ hexane $)$. Spectral data for 6a: ${ }^{1} \mathrm{H}-\mathrm{NMR}$ $\left(\mathrm{CDCl}_{3}, 300 \mathrm{MHz}\right) \delta=0.79(\mathrm{t}, \mathrm{J}=7.1 \mathrm{~Hz}, 3 \mathrm{H}), 1.16(\mathrm{t}, \mathrm{J}=7.1 \mathrm{~Hz}, 3 \mathrm{H}), 1.22-1.40(\mathrm{~m}, 3 \mathrm{H}), 1.40-$ $1.50(\mathrm{~m}, 2 \mathrm{H}), 2.04-2.10(\mathrm{~m}, 1 \mathrm{H}), 2.49(\mathrm{~d}, \mathrm{~J}=6.0 \mathrm{~Hz}, 1 \mathrm{H}), 4.08(\mathrm{q}, \mathrm{J}=7.1 \mathrm{~Hz}, 2 \mathrm{H}) ;{ }^{13} \mathrm{C}\left(\mathrm{CDCl}_{3}, 75\right.$ MHz) $\delta=13.46,13.99,20.73,29.60,34.27,38.31,60.97,170.73$; IR (thin film, cm ${ }^{-1}$ ) 3269(br), 2963(m), 1728(s), 1196(s); mass spectrum, m/z (\% rel intensity) $157 \mathrm{M}^{+}(<1), 128$ (31), 68 (29), 54 (100), 41 (38). Anal calcd for $\mathrm{C}_{8} \mathrm{H}_{15} \mathrm{NO}_{2}$ : C, 61.12; H, 9.62; N, 8.91. Found: C, 61.17; H, 10.09; N, 8.87. 


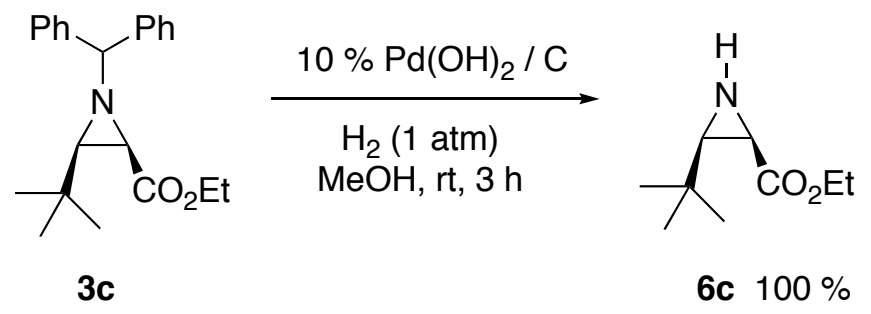

cis-(2R)-Carboxyethyl-(3R)-1,1-dimethylethylaziridine (6c): This reaction was performed with the general procedure and the change in workup noted for 6a. Colorless liquid (will evaporate under high vacuum $) . \mathrm{R}_{f}=0.10\left(1: 1 \mathrm{Et}_{2} \mathrm{O} /\right.$ hexane $) . \quad$ Spectral data for $\mathbf{6 c}:{ }^{1} \mathrm{H}-\mathrm{NMR}\left(\mathrm{CDCl}_{3}, 300\right.$ MHz) $\delta=0.89(\mathrm{~s}, 9 \mathrm{H}), 1.23(\mathrm{t}, \mathrm{J}=6.9 \mathrm{~Hz}, 3 \mathrm{H}), 1.48(\mathrm{br}, \mathrm{s}, 1 \mathrm{H}), 2.09(\mathrm{~d}, \mathrm{~J}=6.1 \mathrm{~Hz}, 1 \mathrm{H}), 2.61(\mathrm{~d}, \mathrm{~J}$ $=6.6 \mathrm{~Hz}, 1 \mathrm{H}), 4.04-4.23(\mathrm{~m}, 2 \mathrm{H}) ;{ }^{13} \mathrm{C}\left(\mathrm{CDCl}_{3}, 75 \mathrm{MHz}\right) \delta=14.00,27.42,31.46,35.25,47.36$, 60.98, 170.20; IR (thin film, $\mathrm{cm}^{-1}$ ) 3276(w), 2957(w), 1738(s), 1202(s), 1032(s); mass spectrum, $\mathrm{m} / \mathrm{z}$ (\% rel intensity) $171 \mathrm{M}^{+}(<1), 156$ (43), 114 (30), 97 (63), 82 (100), 70(88), 55 (63). Anal. calcd for $\mathrm{C}_{9} \mathrm{H}_{17} \mathrm{NO}_{2}: \mathrm{C}, 63.13 ; \mathrm{H}, 10.01 ; \mathrm{N}, 8.18$. Found: C, 63.10; H, 9.94; N, 8.32.

\section{General experimental procedure for the deprotection via ozonolysis illustrated for the azirdine 3e.}

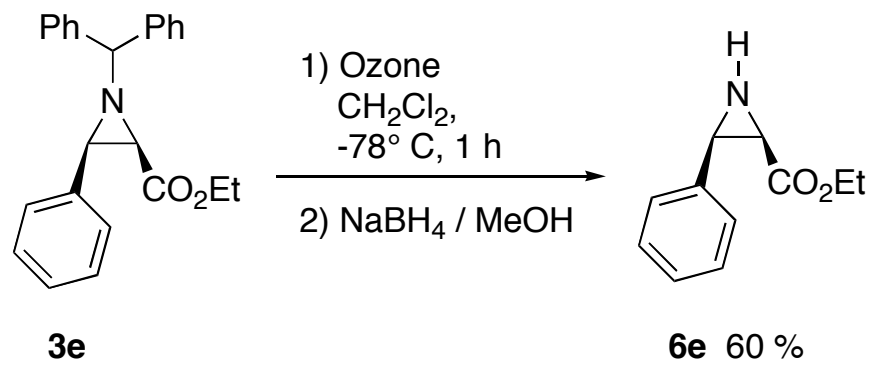

cis-(2R)-Carboxyethyl-(3R)-benzylaziridine (6e): A $250 \mathrm{~mL}$ flame-dried 3-necked round bottom flask was equipped with a $\mathrm{CaCl}_{2}$ drying tube and a glass tube for ozone inlet and then filled with argon. To the flask was added $357 \mathrm{mg}$ cis-N-benzylhydryl-2-carboxyethyl-3-phenylaziridine 3e $(1 \mathrm{mmol})$ and $100 \mathrm{~mL} \mathrm{CH}_{2} \mathrm{Cl}_{2}$. The flask was then immersed in a dry ice-acetone cooling bath at $-78^{\circ} \mathrm{C}$ while nitrogen was bubbled through the solution. The nitrogen purge was stopped and then ozone was bubbled through the stirring solution until the reaction was complete $(\sim 3 \mathrm{~h})$. Upon completion, the addition of ozone was discontinued and then the solution was purged with nitrogen for $5 \mathrm{~min}$ to remove unreacted ozone (the color of the solution changed from blue to colorless). The reaction was quenched by the addition of 10 equiv. of methanolic sodium borohydride and then allowed to gradually warm up to r.t, followed by addition of $50 \mathrm{~mL}$ deionized water. The reaction 
mixture was then extracted with $\mathrm{CH}_{2} \mathrm{Cl}_{2}$, dried with $\mathrm{MgSO}_{4}$, filtered and the filtrate was concentrated on a rotary evaporator and the resulting oil was purified by flash chromatography (15 $\mathrm{mm} \times 300 \mathrm{~mm}$ silica gel, 1:1 ether/hexane) to afford 6e as white solid in $60 \%$ yield (114 mg) White solid, mp: $67-68{ }^{\circ} \mathrm{C} ; \mathrm{R}_{f}=0.13\left(1: 1 \mathrm{Et}_{2} \mathrm{O} /\right.$ hexane). Spectral data for $6 \mathrm{e}:{ }^{1} \mathrm{H}-\mathrm{NMR}\left(\mathrm{CDCl}_{3}\right.$, $300 \mathrm{MHz}) \delta=0.99(\mathrm{t}, \mathrm{J}=7.1 \mathrm{~Hz}, 3 \mathrm{H}), 1.87(\mathrm{br} \mathrm{s}, 1 \mathrm{H}), 3.00(\mathrm{~d}, \mathrm{~J}=6.1 \mathrm{~Hz}, 1 \mathrm{H}), 3.47(\mathrm{~d}, \mathrm{~J}=6.1 \mathrm{~Hz}$, 1H), 3.90-4.00 (m, 2H), 7.24.7.33 (m, 5H); ${ }^{13} \mathrm{C}\left(\mathrm{CDCl}_{3}, 75 \mathrm{MHz}\right) \delta=13.90,29.65,37.14,61.07$, 127.47, 127.62, 128.01, 134.80, 169.02; IR (thin film, $\mathrm{cm}^{-1}$ ) 3218(w), 1738(s), 1186(s), 698(s); mass spectrum, $m / z$ (\% rel intensity) $191 \mathrm{M}^{+}(<1), 162$ (58), 118 (100), 104 (12), 91 (100), 88 (21), 77 (8). Anal calcd for $\mathrm{C}_{11} \mathrm{H}_{13} \mathrm{NO}_{2}$ : C, 69.09; H, 6.85; N, 7.32. Found: C, 68.74; H, 6.91; N, 7.25.

All of the reactions were carried out on racemic aziridines $\mathbf{3}$ except for compound $\mathbf{3 e}$. A sample of 3e that was $98 \%$ ee was deprotected to give $6 \mathbf{e}$ which was found to be $97 \%$ ee. The optical purity of 6e was determined by HPLC analysis on a CHIRALCEL OD-H column with a 90:10 mixture of hexane/2-propanol at $228 \mathrm{~nm}$ with a flow rate of $0.7 \mathrm{~mL} / \mathrm{min}$. Retention times: $\mathrm{R}_{\mathrm{t}}=6.16$ $\min \left(\right.$ minor) and $\mathrm{R}_{\mathrm{t}}=9.47$ min (major). $[\alpha]_{23 \mathrm{D}}=-12.4(\mathrm{c}=1$ in EtOAc) on material that was $99 \%$ ee.

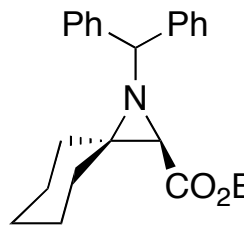

3d

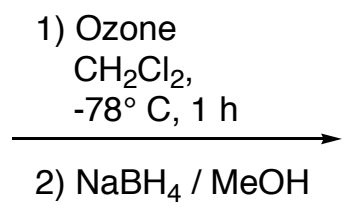

2) $\mathrm{NaBH}_{4} / \mathrm{MeOH}$

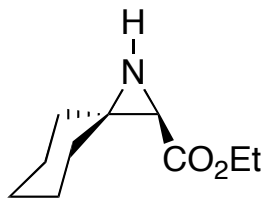

6d $40 \%$

1-Aza-spiro[2.5] octane-2-carboxylic acid ethyl ester (6d): $R_{\mathrm{f}}=0.06$, yield $=40 \%$. Spectral data for $6 \mathrm{~d}:{ }^{1} \mathrm{H}\left(300 \mathrm{MHz}, \mathrm{CDCl}_{3}\right) \delta 1.2(\mathrm{t}, J=7.0 \mathrm{~Hz}, 3 \mathrm{H}), 1.2-1.8(\mathrm{~m}, 10), 2.5(\mathrm{~s}, 1 \mathrm{H}), 2.95$ (bs, $1 \mathrm{H}), 4.23(\mathrm{q}, J=7.0 \mathrm{~Hz}, 2 \mathrm{H}) ;{ }^{13} \mathrm{C}\left(75 \mathrm{MHz}, \mathrm{CDCl}_{3}\right): 14.28,25.10,25.46,25.59,29.54,29.66$, 36.60, 41.87, 61.20, 170.45; IR: 3362, 2928, 2855, 1740, 1635, 1194; HRMS (FAB) calcd for $\mathrm{C}_{10} \mathrm{H}_{18} \mathrm{NO}_{2}^{+} \mathrm{m} / \mathrm{z} 184.1332(\mathrm{M}+1)$, measd 184.1337. 


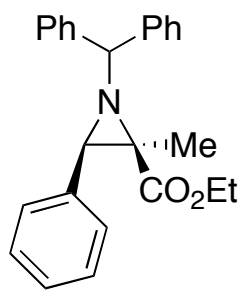

$3 f$

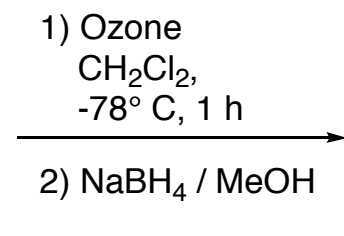

2) $\mathrm{NaBH}_{4} / \mathrm{MeOH}$

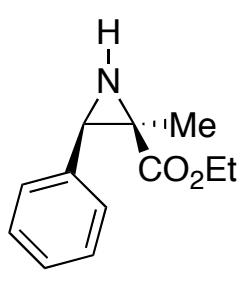

6f $50 \%$

cis-1H-2-Carboxyethyl-2-methyl-3-phenyl aziridine (6f): $\mathrm{R}_{\mathrm{f}}=0.20$ (1:1 hexanes: $\left.\mathrm{Et}_{2} \mathrm{O}\right)$. Yield $50 \%$. Spectral data for 6f: ${ }^{1} \mathrm{H}\left(300 \mathrm{MHz}, \mathrm{CDCl}_{3}\right) \delta 0.94(\mathrm{t}, \mathrm{J}=7.1 \mathrm{~Hz}, 3 \mathrm{H}), 1.65(\mathrm{~s}, 3 \mathrm{H})$, 1.94 (br s, 1H), 3.23 (s, 1H), 3.91 (q, J = 7.1 Hz, 2H), 7.2-7.4 (bs, 5H); ${ }^{13} \mathrm{C}\left(75 \mathrm{MHz}, \mathrm{CDCl}_{3}\right) \delta$ 13.82, 20.35, 42.99, 47.56, 61.23, 127.28, 127.47, 127.85, 127.92, 128.01, 135.49, 170.78; IR: 3303, 2980, 2936, 1729, 1197, 698; HRMS (FAB) calcd for m/z 205.1102 (M+), measd 205.1097.

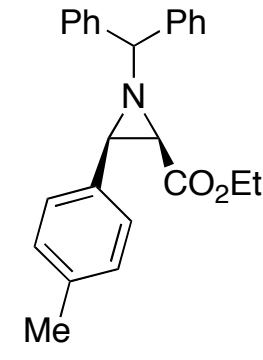

$3 g$

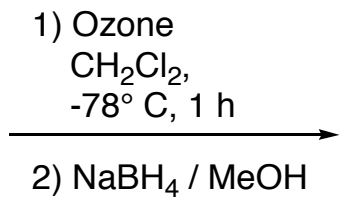

2) $\mathrm{NaBH}_{4} / \mathrm{MeOH}$

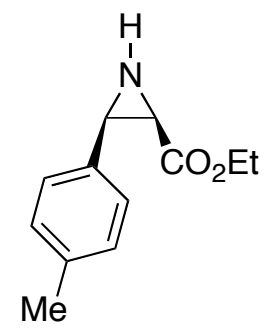

$6 g 51 \%$

cis-1H-2-Carboxyethyl-3-(4-tolyl)-aziridine (6g): White solid, $\mathrm{mp}=54-55^{\circ} \mathrm{C} . \mathrm{R}_{\mathrm{f}}=0.14$ (1:1 hexanes: $\left.\mathrm{Et}_{2} \mathrm{O}\right)$, yield $=51 \%$. Spectral data for $\mathbf{6 g}:{ }^{1} \mathrm{H}\left(300 \mathrm{MHz}, \mathrm{CDCl}_{3}\right) \delta 1.05(\mathrm{t}, J=7.0$ $\mathrm{Hz}, 3 \mathrm{H}), 1.75$ (bs, 1H), 2.30 (s, 3H), 2.96 (d, $J=6.36 \mathrm{~Hz}, 1 \mathrm{H}), 3.43$ (d, J = 6.4 Hz, 1H), 3.98 (m, $2 \mathrm{H}), 7.10(\mathrm{~d}, J=8.1 \mathrm{~Hz}, 2 \mathrm{H}), 7.21(\mathrm{~d}, J=8.05 \mathrm{~Hz}, 2 \mathrm{H}) ;{ }^{13} \mathrm{C}\left(75 \mathrm{MHz}, \mathrm{CDCl}_{3}\right) \delta 13.90,21.05$, 37.03, 39.85, 61.008, 127.28, 128.67, 131.69, 137.22, 169.11; IR: 3310, 2984, 1734, 1383, 1200, 1028, 833; HRMS (FAB) calcd for $\mathrm{C}_{12} \mathrm{H}_{16} \mathrm{NO}_{2}{ }^{+} \mathrm{m} / \mathrm{z} 206.1175(\mathrm{M}+1)$, measd 206.1177. 


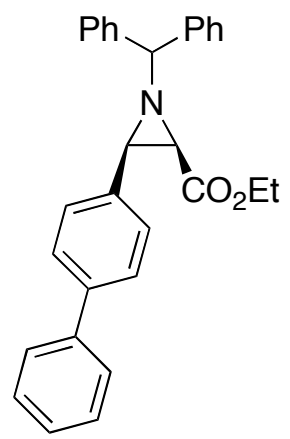

3h

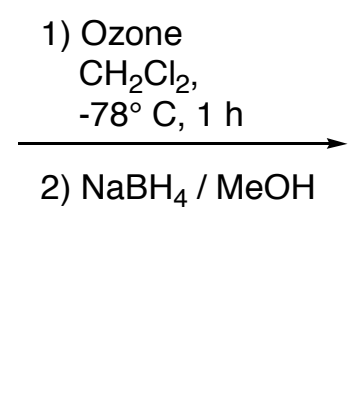

$\mathrm{CH}_{2} \mathrm{Cl}_{2}$

2) $\mathrm{NaBH}_{4} / \mathrm{MeOH}$

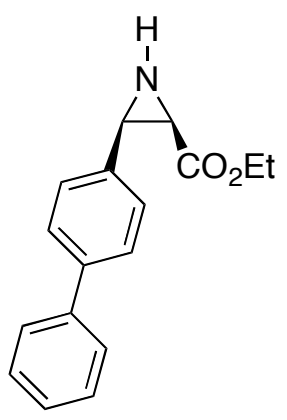

6h $60 \%$

cis-1H-2-Carboxyethyl-3-biphenyl-aziridine (6h): White solid, $\mathrm{mp}=61-62^{\circ} \mathrm{C}, \mathrm{R}_{\mathrm{f}}=0.07$ (1:1 hexane: $\left.\mathrm{Et}_{2} \mathrm{O}\right)$, yield $=60 \%$. Spectral data for $\mathbf{6 h}:{ }^{1} \mathrm{H}\left(300 \mathrm{MHz}, \mathrm{CDCl}_{3}\right) \delta 1.0(\mathrm{bs}, 3 \mathrm{H}), 1.78$ (bs, 1H), 3.0 (bs, 1H), 3.45 (bs, 1H), $4.0(\mathrm{bs}, 2 \mathrm{H}), 7.2-7.62(\mathrm{~m}, 9 \mathrm{H}) .{ }^{13} \mathrm{C}\left(75 \mathrm{MHz}, \mathrm{CDCl}_{3}\right) \delta 13.95$, 37.34, 40.68, 61.07, 126.70, 126.97, 127.27, 127.29, 127.92, 128.69, 133.94, 140.54, 169.02; IR: 3310, 2982, 1734, 1487, 1204, 1030, 760, 698; HRMS (FAB) calcd for $\mathrm{C}_{17} \mathrm{H}_{18} \mathrm{NO}_{2}^{+} \mathrm{m} / \mathrm{z} 268.1332$ $(\mathrm{M}+1)$, measd 268.1341 .

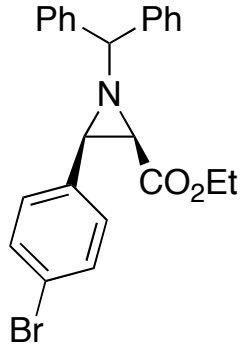

3i

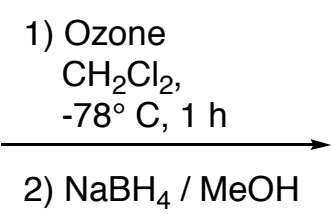

2) $\mathrm{NaBH}_{4} / \mathrm{MeOH}$

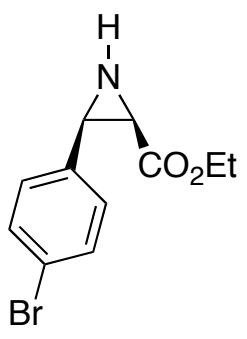

6i $40 \%$

cis-(2R)-Carboxyethyl-(3R)-p-bromobenzylaziridine (6i): White solid, $\mathrm{mp}=78.5-79{ }^{\circ} \mathrm{C} ; \mathrm{R}_{f}=$ $0.12\left(1: 1 \mathrm{Et}_{2} \mathrm{O} / \mathrm{hexane}\right)$. Spectral data for $\mathbf{6 i}:{ }^{1} \mathrm{H}-\mathrm{NMR}\left(\mathrm{CDCl}_{3}, 300 \mathrm{MHz}\right) \delta=1.02(\mathrm{t}, \mathrm{J}=7.1 \mathrm{~Hz}$, 3H), 1.67 (br s, 1H), 3.01 (d, J = 6.6 Hz, 1H), 3.40 (d, J = 6.4 Hz, 1H), 3.93-3.98 (m, 2H), 7.20 (d, J $=8.5 \mathrm{~Hz}, 2 \mathrm{H}), 7.41(\mathrm{~d}, \mathrm{~J}=8.5 \mathrm{~Hz}, 2 \mathrm{H}) ;{ }^{13} \mathrm{C}\left(\mathrm{CDCl}_{3}, 75 \mathrm{MHz}\right) \delta=13.88,37.05,38.98,61.06$, 121.45, 129.18, 130.98, 133.85, 168.62; IR (thin film, $\mathrm{cm}^{-1}$ ) 3222(br s), 1734(vs), 1194(vs), 1170(s), 843(w); mass spectrum, $m / z$ (\% rel intensity) $271 \mathrm{M}^{+}\left({ }^{81} \mathrm{Br}, 2\right), 269 \mathrm{M}^{+}\left({ }^{79} \mathrm{Br}, 2\right), 226\left({ }^{81} \mathrm{Br}, 8\right), 224$ $\left({ }^{79} \mathrm{Br}, 11\right), 197\left({ }^{81} \mathrm{Br}, 100\right), 195\left({ }^{79} \mathrm{Br}, 100\right), 116(96), 89(97), 88$ (21), 77 (13), 63 (26). Anal calcd for $\mathrm{C}_{11} \mathrm{H}_{12} \mathrm{BrNO}_{2}: \mathrm{C}, 48.91 ; \mathrm{H}, 4.48 ; \mathrm{N}, 5.19$. Found: $\mathrm{C}, 48.88 ; \mathrm{H}, 4.50 ; \mathrm{N}, 5.13$. 